\title{
Correlation among Non-radiological Measurements for Thoracic Kyphosis
}

\author{
Sung-hoon Jung, PT, Ph.D ${ }^{1}$; Ui-jae Hwang, PT, Ph.D ${ }^{1}$; Jun-hee Kim, PT, Ph.D ${ }^{1}$; \\ Sung-min Ha, PT, Ph.D²; Oh-yun Kwon, PT, Ph.D ${ }^{1}$ \\ ${ }^{1}$ Physical Therapy, College of Health Science, Yonsei University, Wonju, South Korea \\ ${ }^{2}$ Department of Physical Therapy, College of Health Science, Sangji University, South Korea
}

Background Although there are many measurements for thoracic kyphosis using nonradiological, correlation between the continuous measurement and selective measurement is unclear.

Purpose The purpose of this study is to investigate the correlation among Spinal Mouse (continuous measurement), Flexicurve and Arcometer (selective measurement).

Study design Correlation study

Methods 89 healthy participants were measured by non-radiological measurement tools (Spinal Mouse, Flexicurve and Arcometer) for thoracic kyphosis.

Results The correlation between the Spinal mouse and Flexicurve measurements of thoracic kyphosis was good ( $\mathrm{ICC}=0.53,95 \% \mathrm{CI}=0.37-0.67$ ). And the correlation between the Spinal Mouse and Arcometer measurements of thoracic kyphosis was good (ICC $=0.58,95 \%$ CI $=0.42$ 0.70). The mean difference between the Spinal Mouse and Arcometer was 12.70 degree (difference between Spinal Mouse and Arcometer), between the Spinal mouse and Flexicurve was 23.83 degree (difference between Spinal Mouse and Flexicurve).

Conclusions While Flexicurve, Arcometer are highly correlated to the Spinal Mouse, they have poor agreement. Therefore, physical therapists should take caution when interpreting its results.

Key words Correlation; Measurement; Non-radiological; Thoracic kyphosis.

JMST

2020; 4(1): 1-5

Published Online

Jun 30, 2020

PISSN 2635-8573 eISSN 2635-8581

Article History

Received 02 May 2020

Revised 12 May 2020

(1st)

Revised 19 May 2020

(2nd)

Accepted 20 May 2020

\section{CONTACT}

kwonoy@yonsei.ac.kr Oh-yun Kwon,

Physical Therapy,

College of Health Science,

Yonsei University,

Wonju, South Korea

This is an Open-Access article distributed under the terms of the Creative Commons ribution Non-Conmons Attribution Non-Commercial License (itt.//cativecommons. org/icenses by-ne/4.0) which permits unrestricted non-commercial use, distribution, and reproduction in any medium, provided the original work is properly cited.

\section{INTRODUCTION}

Thoracic hyperkyphosis is greater than 40 degrees of a thoracic cobb angle in sagittal plane. ${ }^{1}$ The curvature of the thoracic vertebrae generally increases with age, and in recent years, the hyperkyphosis is frequently observed between the 20 s and 50s while using digital devices such as computers and smartphones. ${ }^{2-5}$ Thoracic hyperkyphosis reduces balance and increases the risk of falls. ${ }^{6}$ In addition, musculoskeletal problems such as neck pain, shoulder pain, and back pain are also associated with thoracic hyperkyphosis. ${ }^{7-9}$

It is important for physical therapist to measure thoracic kyphosis because thoracic hyperkyphosis have negative health consequences. ${ }^{10}$ The Cobb angle calculated by sagittal plane spinal radiograph is considered the gold standard in thoracic kyphosis measurements. ${ }^{11}$ However, the limitations of radiographic measurements are cost, portability limitations, time consuming, and exposure to ionizing radiation. ${ }^{12,13}$ For this reason, non-radiological methods are preferred in clinical settings, and Spinal Mouse, Flexicurve and Arcometer has previously demonstrated excellent level of validity and intra-inter-rater reliability in previous studies. ${ }^{14}$ These devices have the advantage of being portable without being exposed to ionizing radiation.

Non-radiological methods are skin-surface devices. ${ }^{14,15}$ The Spinal Mouse measures the thoracic curve continuously throughout the thoracic spine, and the Flexicurve and Arcometer calculates the thoracic curve by placing the tool 
at a selected location on the thoracic spine. ${ }^{14}$ If the correlation between the value calculated through continuous measurement and calculated through selective measurement is demonstrated, the accuracy and correlation of the measured values with each tool can be understood and applied to therapy. Therefore, the purpose of this study was to investigate the correlation among Spinal mouse, Flexicurve and Arcometer.

\section{METHOD}

\section{Participants}

People who thought they had thoracic hyperkyphosis were recruited as subjects between 18 and 50 years. Exclusion criteria were (1) participants with scoliosis, (2) a history of spinal column fracture, (3) spinal tumors and related malignancies, (3) congenital spinal anomalies, (4) cancer, and (5) rheumatoid arthritis. Before the experiment, the participants were explained by all procedures of the experimental process. Each provided and signed informed consent on a form approved by the Yonsei University, Mirae Campus, Institutional Review Board (1041849-201901BM-019-01).

\section{Instrumentation}

\section{1) Spinal mouse}

Sagittal spinal curvatures were measured in the standing position at staring straight ahead using a Spinal Mouse system (Idiag, Fehraltdorf, Switzerland). The Spinal Mouse has accelerometers that record change of inclination and intersegmental distance of spinous processes. The device contains two rolling wheels follow the spinous processes of the spine, and the data are transferred from the device to a computer (sampling frequency of approximately $150 \mathrm{~Hz}$ ). ${ }^{16}$ This data is used to calculate the relative angles between the vertebras and total angle of sagittal plane with using Spinal Mouse software. For global spinal angles, the device is a reliable and valid device. ${ }^{17-19}$

\section{2) Flexicurve}

The Flexicurve kyphosis angle was measured using a Flexicurve. ${ }^{10,20}$ The cephalic end of the Flexicurve was placed at $\mathrm{C} 7$ and it was molded to the contour of the thoracic spine in the caudal direction. The Flexicurve was then carefully transferred to paper. Next, a photograph of the Flexicurve imprint was captured using Smartphone camera (Iphone 8, Apple Inc, California). Using a tripod, the camera was parallel to the floor and fixed above $1 \mathrm{~m}$. The Flexicurve angle was analysis using ImageJ (National Institutes of Health, Bethesda, MD) and calculated according to the previous study. ${ }^{10,20}$ The validity and reliability of the measurements from the Flexicurve method have been demonstrated in previous studies. ${ }^{21-25}$

\section{3) Arcometer}

An Arcometer is a method of inferring a thoracic kyphosis angle by measuring several lengths. ${ }^{26}$ The validity and reliability of the measurements from the Arcometer method have been demonstrated by D'Osualdo (1997). In this study, a modified Arcometer manufactured by our team was used (Figure 1A). The modified Arcometer is a tool consisting of a long bar and two smaller, perpendicular bars.

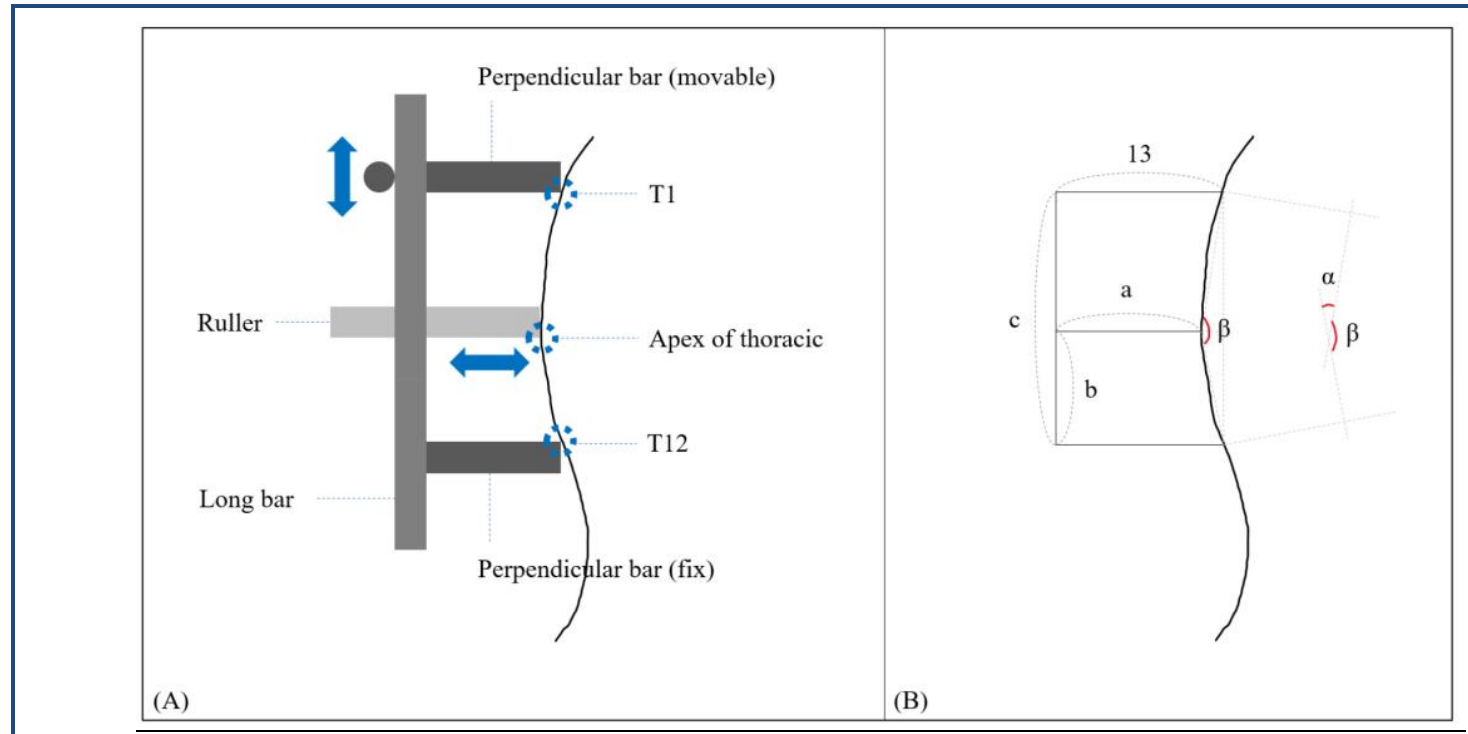

Figure 1. The mimetic diagram of modified Arcometer. 
Each bar is marked with millimeters. The first perpendicular bar is fixed at one end; the second, movable on a single axis. For measuring thoracic kyphosis, the first bar is placed at $\mathrm{T} 1$ and the second bar is placed at T12. Then, the ruler is pierced through a long bar at the apex between T1 and T12 (Figure 2). The tool can provide the three lengths and the thoracic kyphosis angle was calculated according to the following formula (Figure 1B): $\alpha=\pi-\cos ^{-1}\left(\frac{d^{2}+e^{2}-c^{2}}{2 d e}\right)$, $\left(d=\sqrt{b^{2}+\left(13-a^{2}\right)}, e=\sqrt{(c-b)^{2}+(13-a)^{2}}\right)$

\section{Statistical analysis}

The statistical analyses were performed using the statistical software package Statistical Package for the Social Sciences (SPSS) version 20.0 (SPSS Inc., Chicago, IL), and the level of statistical significance was set at $p<0.05$. The Kolmogorov-Smirnov test was used to assess the homogeneity of variance of the Arcometer and Spinal mouse measurement $(p>0.05)$. The correlation among the non-radiological measurement for thoracic kyphosis was assessed by calculating the ICC $[3,1]$ ICC values $>0.75$ were considered excellent; $0.40-0.75$ indicated fair to good; and $0.00-0.40$ indicated poor. ${ }^{27}$ Bland-Altman limits of agreement analysis was used to assess the relative agreement between measurements (Spinal Mouse-Flexicurve and Spinal Mouse-Arcometer). ${ }^{28}$

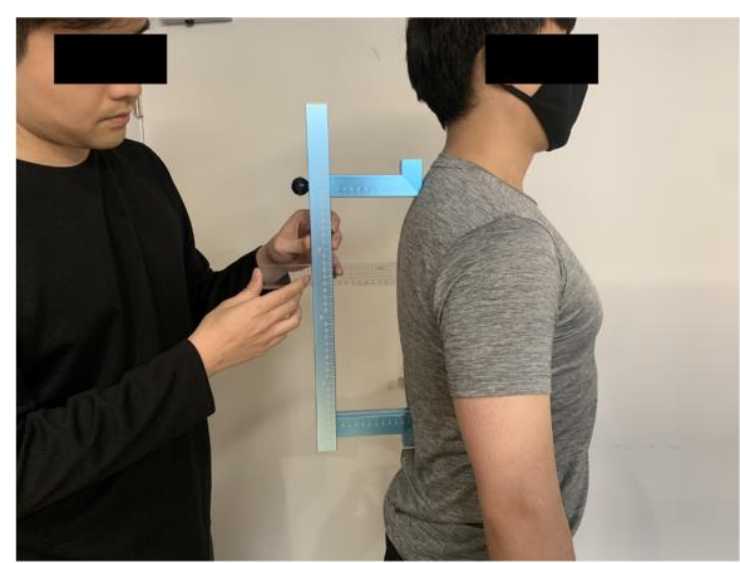

Figure 2. The modified Arcometer.

\section{RESULT}

A total of 89 subjects participated in the study analysis due to loss of data among 5 of the 94 recruited. Eighty-eight subjects (age $=35.25 \pm 6.71$ years; height $=169.37 \pm 7.34 \mathrm{~cm}$; mass $=71.31 \pm 15.24 \mathrm{~kg}$ ) participated in this study. Table 1 displays a description of the thoracic kyphosis values obtained from the Spinal Mouse, Flexicurve and Arcometer.

The correlation between the Spinal mouse and Flexicurve measurements of thoracic kyphosis was good ( $\mathrm{ICC}=0.53$, $95 \% \mathrm{CI}=0.37-0.67)$. And the correlation between the Spinal Mouse and Arcometer measurements of thoracic kyphosis was good $(\mathrm{ICC}=0.58,95 \% \mathrm{CI}=0.42-0.70)$. The correlation between the Arcometer and Flexicurve measurements of thoracic kyphosis was good $(\mathrm{ICC}=0.42,95 \% \mathrm{CI}=0.24$ 0.58). The mean difference between the Spinal Mouse and Arcometer was $12.70^{\circ}$ (Spinal Mouse-Arcometer), between the Spinal mouse and Flexicurve was $23.83^{\circ}$ (Spinal Mouse-Flexicurve) (Bland-Altman plots) (Figure 3, 4). In addition, there were few outliers beyond two SDs of the mean difference. Therefore, the Arcometer tended to consistently produce measurements that were approximately $12.7^{\circ}$ lower than those of the Spinal Mouse and Flexicurve tended to consistently produce measurement that were approximately $23.83^{\circ}$ lower than those of the Spinal Mouse.

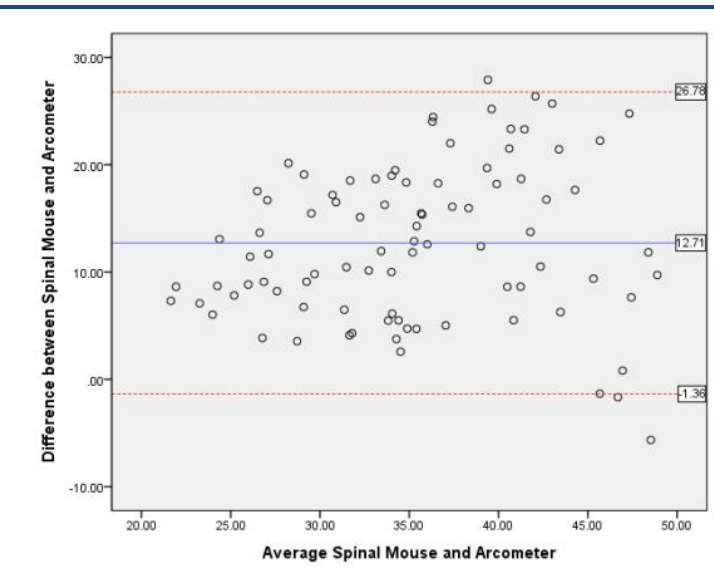

Figure 3. Bland-Altman plot showing the relative agreement between Spinal Mouse and Arcometer of measurement with $95 \%$ limits of agreement.

Table 1. Description of the thoracic kyphosis values obtained from the Spinal Mouse, Flexicurve and Arcometer

\begin{tabular}{ccccc}
\hline & Mean $\left({ }^{\circ}\right)$ & Standard deviation $\left(^{\circ}\right)$ & Minimum $\left({ }^{\circ}\right)$ & Maximum $\left({ }^{\circ}\right)$ \\
\hline Spinal Mouse & 41.65 & 8.24 & 25.3 & 59.7 \\
Flexicurve & 17.82 & 5.39 & 4.05 & 29.14 \\
Arcometer & 28.96 & 7.40 & 17.62 & 51.35 \\
\hline
\end{tabular}




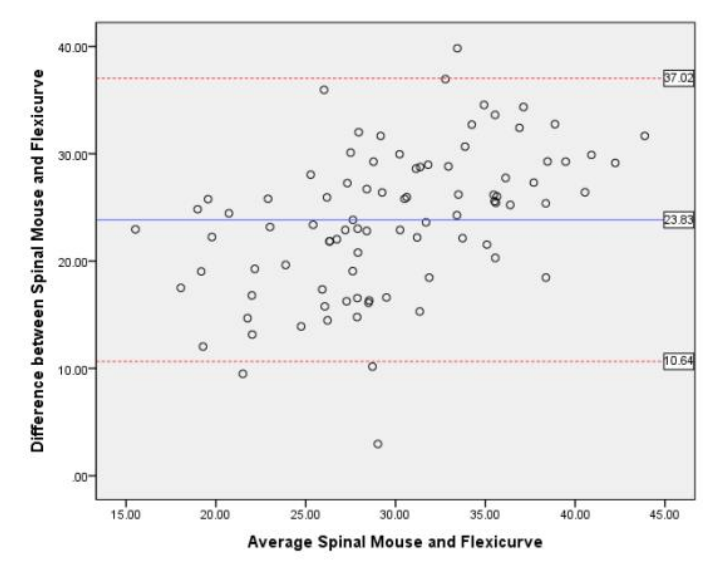

Figure 4. Bland-Altman plot showing the relative agreement between Spinal Mouse and Flexicurve of measurement with $95 \%$ limits of agreement.

\section{DISCUSSION}

This study investigated the correlation between nonradiological measurements. To reduce exposure to ionizing radiation in clinical settings, non-radiological methods are often used to measure thoracic kyphosis in patients. ${ }^{12,13}$ Since there are a variety of non-radiological methods to measure thoracic kyphosis, the results of the correlation among nonradiological methods will help clinicians treat patients.

In the previous study, the range of the Cobb angle measured by the radiography was $20.3^{\circ}-66.3^{\circ} .{ }^{29}$ Among the methods used in this study, the range of the thoracic kyphosis measured by the Spinal Mouse $\left(25.3^{\circ}-59.7^{\circ}\right)$ had the most similar range to the previous study measured by the radiography. However, the Flexicurve and Arcometer showed the range of $4.05^{\circ}-29.14^{\circ}$ and $17.62^{\circ}-51.35^{\circ}$, respectively, showing a large difference from the range in the previous study measured by the radiography. Since the Spinal Mouse is a continuous measurement method, it may have a range similar to that of the radiography than the selective measurement method of Flexicurve and Arcometer. ${ }^{14}$

The average difference between Spinal Mouse and Flexicurve is greater than the average difference between Spinal Mouse and Arcometer. For measurement of the thoracic kyphosis, the Arcometer places the tool on the thoracic spine in the standing and simultaneously measures the length value, while the Flexicurve places the tool on the thoracic spine, tracks the shape, takes a picture, and measures it through the program. ${ }^{20}$ The process using Flexicurve is more complicated than the process using Arcometer, and the measurement and analysis mistakes that can occur in this process can make an average difference.

Our study has several limitations. First, we enrolled only healthy, relatively young subjects (35.25 \pm 6.71 years). Thus, our findings cannot be generalized to any patient population, to subjects with old-age. Second, we measured single measurement. Repeated measurements may have yielded different results.

\section{CONCLUSIONS}

The selective measurements (Flexicurve, Arcometer) are highly correlated to the continuous measurement (Spinal Mouse). However, they have poor agreement (mean difference: Arcometer $12.70^{\circ}$, Flexicurve $23.83^{\circ}$ ). Therefore, physical therapists should take caution when interpreting its results.

\section{Key Points}

Question What about the correlation among non-radiological measurements for thoracic kyphosis?

Findings The correlation among non-radiological measurements were good. The mean difference was 12.70 degree (difference between Spinal Mouse and Arcometer), and was 23.83 degree (difference between Spinal Mouse and Flexicurve).

Meaning While Flexicurve, Arcometer is highly correlated to the Spinal Mouse, they have poor agreement.

\section{Article information}

Conflict of Interest Disclosures: None.

Funding/Support: The authors acknowledge financial and administrative support provided by Yonsei University Research Fund (2018-51-0001 and 2018-51-0477).

Acknowledgment: None.

Ethic Approval: 1041849-201901-BM-019-01.

\section{REFERENCES}

1. Bansal S, Katzman WB, Giangregorio LM. Exercise for improving age-related hyperkyphotic posture: a systematic review. Arch Phys Med Rehabil. 2014;95(1):129-140.

2. Fon GT, Pitt MJ, Thies Jr AC. Thoracic kyphosis: range in normal subjects. AJR Am J Roentgenol. 1980;134(5): 979-983.

3. Takahashi T, Ishida K, Hirose D, et al. Trunk deformity is associated with a reduction in outdoor activities of daily living and life satisfaction in community-dwelling older people. Osteoporos Int. 2005;16(3):273-279.

4. Park J-H, Kang S-Y, Lee S-G, Jeon H-S. The effects of smart phone gaming duration on muscle activation and spinal posture: pilot study. Physiother Theory Pract. 2017; 33(8):661-669. 
5. Seidi F, Rajabi R, Ebrahimi I, Alizadeh MH, Minoonejad $\mathrm{H}$. The efficiency of corrective exercise interventions on thoracic hyper-kyphosis angle. J Back Musculoskelet Rehabil. 2014;27(1):7-16.

6. Sinaki M, Brey RH, Hughes CA, Larson DR, Kaufman KR. Balance disorder and increased risk of falls in osteoporosis and kyphosis: significance of kyphotic posture and muscle strength. Osteoporos Int. 2005;16(8): 1004-1010.

7. Griegel-Morris P, Larson K, Mueller-Klaus K, Oatis CA. Incidence of common postural abnormalities in the cervical, shoulder, and thoracic regions and their association with pain in two age groups of healthy subjects. Phys Ther. 1992;72(6):425-431.

8. Gumina S, Di Giorgio G, Postacchini F, Postacchini R. Subacromial space in adult patients with thoracic hyperkyphosis and in healthy volunteers. Chir Organi Mov. 2008;91(2):93-96.

9. Ensrud KE, Black DM, Harris F, Ettinger B, Cummings SR. Correlates of kyphosis in older women. J Am Geriatr Soc. 1997;45(6):682-687.

10. Barrett E, Lenehan B, O'sullivan K, Lewis J, McCreesh $\mathrm{K}$. Validation of the manual inclinometer and flexicurve for the measurement of thoracic kyphosis. Physiother Theory Pract. 2018;34(4):301-308.

11. Harrison DE, Cailliet R, Harrison DD, Janik TJ, Holland B. Reliability of centroid, Cobb, and Harrison posterior tangent methods: which to choose for analysis of thoracic kyphosis. Spine (Phila Pa 1976). 2001; 26(11):e227-e234.

12. de Oliveira TS, Candotti CT, La Torre M, et al. Validity and reproducibility of the measurements obtained using the flexicurve instrument to evaluate the angles of thoracic and lumbar curvatures of the spine in the sagittal plane. Rehabil Res Pract. 2012;2012:186156.

13. Briggs A, Wrigley T, Tully E, Adams P, Greig A, Bennell K. Radiographic measures of thoracic kyphosis in osteoporosis: cobb and vertebral centroid angles. Skeletal Radiol. 2007;36(8):761-767.

14. Barrett E, McCreesh K, Lewis J. Reliability and validity of non-radiographic methods of thoracic kyphosis measurement: a systematic review. Man Ther. 2014;19(1): $10-17$.

15. Zafereo J, Wang-Price S, Brown J, Carson E. Reliability and comparison of spinal end-range motion assessment using a skin-surface device in participants with and without low back pain. J Manipulative Physiol Ther. 2016;39(6):434-442.

16. Livanelioglu A, Kaya F, Nabiyev V, Demirkiran G, Firat T. The validity and reliability of "Spinal Mouse" assess- ment of spinal curvatures in the frontal plane in pediatric adolescent idiopathic thoraco-lumbar curves. Eur Spine J. 2016;25(2):476-482.

17. Guermazi M, Ghroubi S, Kassis M, et al. Validity and reliability of Spinal Mouse to assess lumbar flexion. Ann Readapt Med Phys. 2006;49(4):172-177.

18. Mannion AF, Knecht K, Balaban G, Dvorak J, Grob D. A new skin-surface device for measuring the curvature and global and segmental ranges of motion of the spine: reliability of measurements and comparison with data reviewed from the literature. Eur Spine J. 2004;13(2): 122-136.

19. Post R, Leferink V. Spinal mobility: sagittal range of motion measured with the Spinal Mouse, a new noninvasive device. Arch Orthop Trauma Surg. 2004;124(3): 187-192.

20. Quek J, Pua Y-H, Clark RA, Bryant AL. Effects of thoracic kyphosis and forward head posture on cervical range of motion in older adults. Man Ther. 2013;18(1): 65-71.

21. Milne J, Williamson J. A longitudinal study of kyphosis in older people. Age Ageing. 1983;12(3):225-233.

22. Hart DL, Rose SJ. Reliability of a noninvasive method for measuring the lumbar curve. J Orthop Sports Phys Ther. 1986;8(4):180-184.

23. Simpson S. Evaluation of a flexible ruler technique for measuring lumbar lordosis in the clinical assessment of low back pain. $J$ Soc Occup Med. 1989;39(1):25-29.

24. Tillotson KM, Burton AK. Noninvasive measurement of lumbar sagittal mobility. An assessment of the flexicurve technique. Spine (Phila Pa 1976). 1991;16(1):29-33.

25. Greendale G, Nili N, Huang M-H, Seeger L, Karlamangla A. The reliability and validity of three non-radiological measures of thoracic kyphosis and their relations to the standing radiological Cobb angle. Osteoporos Int. 2011;22(6):1897-1905.

26. D'Osualdo F, Schierano S, Iannis M. Validation of clinical measurement of kyphosis with a simple instrument, the arcometer. Spine (Phila Pa 1976). 1997;22(4): 408-413.

27. Portney L, Watkins M. Foundations of clinical applications to practice. Upper Saddle River, NJ: Prentice Hall; 2008.

28. Bland JM, Altman D. Statistical methods for assessing agreement between two methods of clinical measurement. Lancet. 1986;327(8476):307-310.

29. Edmondston SJ, Christensen MM, Keller S, Steigen LB, Barclay L. Functional radiographic analysis of thoracic spine extension motion in asymptomatic men. $J \mathrm{Ma}$ nipulative Physiol Ther. 2012;35(3):203-208. 